\title{
La predicción afectiva positiva como factor de protección socio- emocional en madres transnacionales antes y después de la reagrupación familiar: relación con la satisfacción con la vida, regulación emocional, soledad social, resiliencia y estrés
}

\section{Positive affective prediction as a factor of social emotional protection in transnational mothers before and after family reunification: relationship with life satisfaction, emotional regulation, social loneliness, resilience and stress}

\author{
Cristina Martínez-Taboada \\ Alicia Albeza \\ Alberto Amutio \\ Gabriela Nicolae \\ Departamento Psicología Social y Metodología de las Ciencias del Comportamiento \\ Universidad del País Vasco (UPV/EHU), España
}

Rec (1 de junio de 2016) Acept (22 de marzo de 2017)

\begin{abstract}
Resumen
La predicción afectiva es un proceso de sobrestimación emocional previo a un acontecimiento. Suele asociarse a altas expectativas y a un coste socioemocional posterior. Para estudiar las diferencias entre las predicciones afectivas antes y después de la reagrupación familiar transnacional, se analizan a 188 madres inmigrantes residentes en España, bien expectantes por reagrupar, bien reagrupadas con sus hijos. Se hipotetiza y confirma la existencia de diferencias estadísticamente significativas entre las mujeres en situación antes y después en relación a satisfacción con la vida, control emocional, resiliencia, estrés y soledad social. Además, se, resalta la función positiva del pronóstico afectivo en el ajuste socio cognitivo de las madres transnacionales. Previamente al evento, éste media entre la satisfacción y la soledad social, y después de la reagrupación, tiene una función mediadora entre la satisfacción vital y el descontrol emocional asociado al encuentro lo que expresa su papel protector en la conciliación familiar.
\end{abstract}

Palabras clave: Predicción afectiva; Reagrupación familiar; Madres latinoamericanas transnacionales.

\begin{abstract}
Affective forecasting is an emotional process of overestimation of the future. Previous studies indicate that if these expectations are excessive and do not coincide with reality they can lead to a high socio emotional cost after the event. In order to analyze the differences in affective predictions before and after transnational reunification 188 immigrant mothers resident in Spain were divided in two groups according to whether or not they had regrouped with their children. Both groups were homogeneous in socio-demographics (age, number of children, education level, employment status, and reasons for migrating). The results confirm the existence of significant differences between emotional experience, before and after reunification. It was found that affective forecasting played a positive role before and after the event for the variables affecting socio-cognitive regulation in both groups (well-being, emotional regulation, resilience and stress), but in different manner during the process of transnational family reunification.

Key words: Affective forecasting; Family reunification; Latin-American transnational mothers.
\end{abstract}

\footnotetext{
Correspondencia: Alberto Amutio Careaga. Facultad de Relaciones Laborales y Trabajo Social. Universidad del País Vasco (UPV/EHU). Barrio Sarriena, s/n. Campus de Leioa, 48940. Teléfono: +349446013117. Fax:+34946012277. Email: alberto.amutio@ehu.eus

Nota: Estudio financiado por el Dpto. de Educación, Universidades e Investigación del Gobierno Vasco en relación a la beca pre doctoral N Ref. BFI09.291. Departamento de Política familiar y Comunitaria del Gobierno Vasco N Ref. 2011.0195
} 


\section{Introducción}

El ser humano presenta una capacidad innata a tener expectativas e ilusiones de futuro. Esta necesidad de predecir se asocia en realidad al anhelo o deseo de confirmar que los eventos traerán bienestar, y para ello se pronostica positivamente cuáles serán los estados afectivos que los acompañarán (Gilbert, 2006). En este sentido, Wilson y Gilbert (2003) diseñan un esquema conceptual denominado "affective forecasting" donde explican que la proyección de los estados emocionales para el futuro, en pos de maximizar el bienestar, influye en las preferencias y comportamientos de las personas. Además augura acontecimientos, dotándolos de significación sobrevalorada en intensidad y duración emocional lo que genera expectativas que luego en la experiencia afectiva real no coinciden una vez que el evento ha sucedido (Gilbert y Wilson 2009).

La familia transnacional está dividida espacialmente, vive al menos en dos lugares manteniendo sus componentes relacionales en la distancia. Un ejemplo típico es el de las madres migrantes separadas de sus hijos. El reencuentro familiar es un evento deseado y está asociado a emociones positivas Elgorriaga, Martínez-Taboada, y Arnoso (2014), así como a un alto grado de idealización producto de fuertes pautas culturales (Graham, 2010) una valoración del país receptor como contexto de oportunidades para sí mismas y para sus hijos (Pérez Grande, 2008), que lleva a sobrevalorar la vida familiar en el país de acogida (Medina, 2011). Sin embargo, la literatura al respecto también indica que en la búsqueda de bienestar, los inmigrantes añaden duelos por la separación de sus familias y amigos. Esta vivencia se asocia a emociones negativas como la percepción subjetiva de soledad (Achotegui, 2004) así como la soledad social derivada de la falta de una red de apoyo estructurada en el nuevo contexto (Weiss, 1972). En el caso de las madres latinoamericanas, el familismo latino constituye uno de los valores culturales centrales (Ayón et al., 2010), que agrega sufrimiento a la separación de los hijos, a la vivencia de soledad y genera necesidades afectivas que inciden en la decisión de reagrupar y en la idealización del vínculo (Lemos y Londoño, 2006).

Wilson y Gilbert (2003) examinan variables que influyen y sesgan la predicción afectiva así como las diferencias emocionales de su impacto. Uno de estos sesgos se deriva de la corrección única de influencias ante el evento anunciado. Por medio de este proceso al momento de predecir se minimizan las dificultades que formarán parte de la experiencia real substrayéndose de posibles dificultades que puedan influir en la vivencia emocional. Dada su congruencia teórica se puede asociar al concepto de evitación cognitiva, entendida como una variable que restringe los elementos que generan incertidumbre, aunque a la larga vuelvan a emerger. Así mismo, los estudios sobre inmigración muestran cuotas de estrés y de descontrol emocional (Martínez-Taboada, Arnoso y Elgorriaga, 2006) que se incrementan con la reagrupación. El modelo de Wilson y Gilbert, $(2003,2005)$ considera que las personas disponen de un sistema inmunológico psicológico que otorga la capacidad de resignificar hechos, ser autoeficaces y encontrar sentido a lo que se vive (Fernandes de Araújo, y Bermúdez, 2015), y el cual se asocia con la acepción de resiliencia. Así y acorde al análisis teórico del "affective forecasting", será necesario medir las variables que inciden en el ajuste sociocognitivo, tanto en las madres antes del reencuentro con sus hijos, como en las madres después del evento de la reagrupación. Esto permitirá conocer la realidad socio emocional de estas mujeres en ambas condiciones de reagrupación

El objetivo del estudio se centra en comprobar la influencia de la predicción afectiva en el proceso de reagrupación transnacional en valencia e intensidad, tanto antes como después de ocurrir el evento. La hipótesis del estudio es que el grupo de madres en situación antes de la reagrupación familiar mostrará un pronóstico afectivo de mayor valencia positiva, intensidad y duración que el de las mujeres en situación después de reagrupadas con sus hijos. Así mismo, se considera que habrá diferencias estadísticamente significativas entre las dos condiciones de madres transnacionales en la percepción de satisfacción con la vida, la regulación emocional, la resiliencia, la evitación, la soledad y el estrés, así como en la relación con la intensidad de la predicción afectiva.

\section{Método}

\section{Procedimiento}

Los contactos con las participantes se obtuvieron por medio de áreas de Inmigración, de Asociaciones de mujeres y de Inmigrantes así como por el sistema bola de nieve. La participación fue voluntaria, explicitando los motivos y garantizando las condiciones de anonimato y de confidencialidad a fin de cumplir con los marcos éticos y legales. 


\section{Participantes}

Muestra no probabilística intencional, compuesta por 188 madres de América Latina gestoras de la reagrupación familiar en el norte de España (País Vasco). La edad de las mujeres comprende de 25 a 55 años, con un promedio de 35 años $(D T=7,15)$. En un $40 \%$ de los casos tienen más de dos hijos, y en un $80 \%$ son menores de edad. En un $53 \%$ de los casos son madres que están solas. En un 51,6\% tienen otros familiares en España. Un 44\% complementaron los estudios medios. El tipo de trabajo que realizan las madres inmigrantes se encuentra vinculado en un $52,5 \%$ a servicio doméstico y/o cuidado de personas, un 31,1\% a hostelería o similares, y un $16,7 \%$ manifiesta estar desempleadas.

\section{Instrumentos}

- Predicción afectiva: escala ad hoc de auto informe con preguntas Likert con 7 opciones de respuesta $(1=$ muy mal, $7=$ excelente) (e.g. “ ¿Cómo se va a sentir cuando reagrupe a sus hijos?”; “CCómo se siente después haber reagrupado a sus hijos?"). Recoge la intensidad y duración antes del evento, y, después de acontecida la reagrupación con los hijos. La mayoría de los estudios de previsión afectiva hace uso de escalas de auto informe que piden a las personas que puntúen antes y después del evento a estudiar (Carlsmith, Wilson y Gilbert, 2008). El alfa de Cronbach $(\alpha)$ fue de 0,80 para nuestra muestra.

- Satisfacción con la vida: Satisfaction With Life Scale (SWLS; Diener, Emmons, Larsen y Griffin, 1985), en la versión adaptada al castellano de Cabañero et al., (2004). El instrumento consta de 5 preguntas, con 7 opciones de respuesta $(1=$ completamente en desacuerdo, $7=$ completamente de acuerdo), (e.g. "Estoy satisfecho con mi vida"). La escala original tiene buena consistencia interna y en nuestra muestra el alfa de Cronbach fue , 82 .

- Afectos positivos y negativos: escala PANAS cuestionario de afecto positivo y negativo de Watson, Clark y Tellegen (1988), adaptada al español por Sandín et al., (1999). El cuestionario incluye 20 ítems tipo Likert 10 para los afectos positivos (e.g. "Animada, Decidida") y 10 para los negativos (e.g.,. "Asustada, Avergonzada"), con 5 opciones de respuesta ( $1=$ ligeramente, $5=$ extremadamente). Tal como lo indican los autores que la adaptan, las propiedades de la escala ha sido comprobada en diversas culturas y países. Además, muchos de los estudios sobre predicción afectiva la utilizan (Wilson y Gilbert, 2003). En la muestra estudiada, la escala muestra una fiabilidad de $\alpha=0.89$ para la subescala afectos positivos, y de $\alpha=0.86$ en la subescala de afectos negativos.

- Resiliencia: Se utilizó la escala de Resiliencia de Wagnild y Young (1993), en sus dimensiones de autoeficacia y sentido en la versión adaptada al castellano de Rodríguez et al., (2009). Las subescalas constan de 15 y 5 ítems tipo Likert, respectivamente (e.g. "He podido atravesar situaciones dificiles porque he experimentado dificultades antes"), con 5 opciones de respuesta ( $1=$ totalmente de acuerdo, $5=$ totalmente en desacuerdo). Presenta buenas propiedades psicométricas, con un $\alpha=$ 0.72 (autoeficacia) y de $\alpha=0.86$ (sentido), para nuestra muestra.

- Percepción de soledad: Test UCLA de Soledad (Russell, Peplau y Cutrona, 1980), en la versión de Borges et al., (2008). La prueba consta de 2 dimensiones: soledad social y emocional, compuestas por 13 ítems tipo Likert, con 5 opciones de respuesta (1=siempre, $5=$ nunca), (e.g. "Me siento solo"). Muestra buenas propiedades psicométricas en la muestra de estudio para los dos factores, con una fiabilidades de $\alpha=0.72$ para la soledad emocional y $\alpha$ $=.83$ para la evaluación subjetiva de la red social.

- Evitación cognitiva: escala de Estrategias de Afrontamiento del Estrés (CAE) (Sandín y Chorot, 2003), utilizando la subescala correspondiente a la dimensión evitación, compuesta por 6 ítems tipo Likert, con 6 opciones de respuesta ( $1=$ nunca, $6=$ siempre), (e.g. "Procuré no pensar en el problema"). Esta dimensión presenta buenas propiedades psicométricas con un alfa de Crombach de $\alpha=0.91$ para nuestra muestra.

- Estrés: Escala de Estrés Percibido de Cohen, Tamarack y Mermelstein (1983), en la versión al castellano de Remor y Carrobles (2001). Se trata de una escala de auto-informe, que evalúa el nivel de estrés percibido en el último mes, compuesta por 14 ítems con 5 opciones de respuesta ( $0=$ nunca, $4=$ muy a menudo), (e.g. "En el último mes, ¿con qué frecuencia se ha sentido nerviosa o estresada?"). Presenta una fiabilidad de $\alpha=0.92$ en nuestra muestra.

- Descontrol emocional: subescala de descontrol emocional del instrumento Dificultad en la Regulación Emocional (Gratz y Roemer, 2004), en la adaptación al castellano de Hervás y Jodar (2008). Esta subescala consta de 9 ítems, con 5 opciones de respuesta $(1=$ casi nunca, $5=$ casi siempre), (eg. "Vivo mis emociones como algo desbordante y fuera de control"). La prueba asociada a Descontrol Emocional presenta buenas propiedades psicométricas con un alfa de Crombach de 0.81 para nuestra muestra. 
Tabla 1. Predicción afectiva antes y después de la reagrupación familiar hijos en España

\begin{tabular}{lccccc}
\hline & $\begin{array}{c}\text { Antes R* } \\
\text { media }\end{array}$ & $\begin{array}{c}\text { Después R* } \\
\text { media }\end{array}$ & $\begin{array}{c}\text { Antes R* } \\
\text { DT }\end{array}$ & $\begin{array}{c}\text { Después R* } \\
\text { DT }\end{array}$ & $t$-Student \\
\hline $\begin{array}{l}\text { Predicción Afectiva de la Reagrupación } \\
\text { Predicción emociones }\end{array}$ & 5.98 & 4.92 & 1.95 & .99 & $P<.000$ \\
$\begin{array}{l}\text { ¿Cómo se siente/sentirá de haber reagrupado hijos? } \\
\text { Predicción intensidad }\end{array}$ & 5.86 & 5.51 & 2.12 & .85 & $P<.145$ \\
$\begin{array}{l}\text { ¿Cuán intensos son/serán esos sentimientos? } \\
\text { Predicción duración }\end{array}$ & 6.09 & 4.48 & 2.02 & 1.47 & $P<.000$ \\
¿Cuánto tiempo durará lo que siente? & 5.99 & 4.76 & 2.09 & 1.35 & $P<.000$ \\
\hline
\end{tabular}

* Antes R: Grupo de madres antes de la reagrupación familia en España.

* Después R: Grupo de madres después de reagrupar hijos en España.

\section{Diseño}

Se trata de un diseño de comparación con grupo estático (Campbell y Standley, 2011). Para robustecer este diseño, se proponen estrategias vinculadas al diseño ex post facto prospectivo simple transversal (Fontes, García, Garriga, Pérez-Llantada y Sarriá, 2010). Se acudió al emparejamiento o apareamiento entre los grupos, es decir se los homogeneizó, en todas las condiciones ambientales, controlando que ambos grupos tengan iguales valores en las variables sociodemográficas (Campbell y Standley, 2011; Fontes et al., 2010). La muestra es homogénea y los grupos de madres antes y después del encuentro familiar son comparables en tanto no se observan diferencias significativas en los grupos de estudio en: edad de la madres $(p=.085)$, cantidad de hijos $\left(\chi^{2}=.785\right)$, edad de los hijos $\left(\chi^{2}=.734\right)$, trabajo actual $\left(\chi^{2}=.349\right)$, nivel de estudios $\left(\chi^{2}=.062\right)$ y motivos por los que migran $\left(\chi^{2}=.334\right)$.

Hablamos de dos condiciones acorde al modelo de Wilson y Gilbert (2003) en el que el grupo antes son aquellas mujeres sin haber acontecido el evento de la reagrupación y el grupo de mujeres después del efecto posterior a la reunificación con los hijos. No se trata de una diferencia temporal sino de condiciones tal como se subdividen los grupos en otros estudios de predicción afectiva (Nielsen, Knutson y Carstensen, 2008).

\section{Resultados}

Se observan (Tabla 1) diferencias estadísticamente significativas $(p=.000)$ entre las mujeres que esperan reagrupar a sus hijos, predicción afectiva del reencuentro sobrevalorado y más elevado, que aquellas que ya tienen la vivencia emocional de estar con sus hijos. A su vez, las mujeres antes de reagrupar a sus hijos muestran emociones más intensas y duraderas, en comparación a la intensidad y duración de las madres ya reagrupadas.

No se advierten diferencias estadísticamente significativas en la satisfacción con la vida y los afectos positivos, entre los grupos de madres antes del rencuentro con sus hijos y madres después de la reagrupación. Así mismo, se observa que existe menos resiliencia, en términos de Autoeficacia y sentido de la situación, en el grupo de mujeres después de reagrupados los hijos en comparación con el grupo de madres antes de reagrupar (Tabla 2).

En la Tabla 3, se observa que el grupo de mujeres después del evento presenta de modo estadísticamente significativo, más estrés, más descontrol emocional, y menos percepción de soledad, tanto emocional, como social, que el grupo antes del evento.

La predicción afectiva aunque muestra diferencias significativas entre antes y después de la reagrupación, se mantiene elevada en los dos grupos con valores por encima del valor medio (tabla 1). El $81 \%$ de las madres realiza una predicción afectiva positiva y elevada de la reagrupación. Para profundizar en su estudio se realizó un análisis de correlaciones con las variables asociadas a la predicción afectiva y posteriormente se procedió a indagar cuales de ellas son las que mejor la explican. Finalmente, se analizó el posible rol mediador del pronóstico afectivo del reencuentro entre las variables que lo predicen. 
Tabla 2. Satisfacción. afecto positivo. resiliencia: comparación entre el grupo de madres antes y después del reencuentro con sus hijos

\begin{tabular}{lccccc}
\hline & $\begin{array}{c}\text { Antes RF* } \\
\text { media }\end{array}$ & $\begin{array}{c}\text { Después RF* } \\
\text { media }\end{array}$ & $\begin{array}{c}\text { Antes RF* } \\
\text { DT }\end{array}$ & Después RF * DT & $t$-Student. \\
\hline Satisfacción & 4.48 & 4.87 & 1.48 & 1.29 & $P<.064$ \\
Afecto pos & 4.13 & 4.08 & .86 & .65 & $P<.668$ \\
Resiliencia & 4.20 & 3.39 & .62 & .82 & $P<.000$ \\
Autoeficacia & 4.39 & 3.34 & .58 & .85 & $P<.000$ \\
Sentido & 4.13 & 3.35 & .74 & .92 & $P<.000$ \\
\hline
\end{tabular}

*Antes RF: grupo de madres antes de la reagrupación familiar en España.

*Después $R F$ : grupo de madres después de reagrupar hijos en España.

Tabla 3. Soledad, evitación, estrés, afectos negativo, descontrol emocional: comparación entre el grupo de madres antes y después del reencuentro con sus hijos

\begin{tabular}{|c|c|c|c|c|c|}
\hline & $\begin{array}{l}\text { Antes RF* } \\
\text { media }\end{array}$ & $\begin{array}{l}\text { Después RF * } \\
\text { media }\end{array}$ & $\begin{array}{c}\text { Antes RF } \\
\text { DT }\end{array}$ & $\begin{array}{c}\text { Después RF } \\
\text { DT }\end{array}$ & t- Student \\
\hline Soledad & 2.83 & 1.98 & .87 & .84 & $P<.000$ \\
\hline Sol. Emocional & 3.59 & 2.22 & 1.07 & 1.04 & $P<.000$ \\
\hline Sol. Social & 2.09 & 1.73 & .84 & .81 & $P<.003$ \\
\hline Evitación & 4.11 & 3.77 & 1.35 & 1.49 & $P<.105$ \\
\hline Estrés & 2.38 & 2.61 & .85 & .62 & $P<.042$ \\
\hline Descontrol & 1.60 & 1.84 & .87 & .71 & $P<.045$ \\
\hline Afecto negativo & 1.99 & 1.80 & .77 & .79 & $P<.101$ \\
\hline
\end{tabular}

*Antes $R F$ : grupo de madres antes de la reagrupación familiar en España.

*Después $R F$ : grupo de madres después de reagrupar hijos en España.

Los análisis de correlación de Pearson, prueban que la predicción afectiva antes del reencuentro tiene una relación positiva con la satisfacción con la vida $(r=, 35)(p=, 01)$ y se vincula negativamente, con la soledad $(r=-, 32)(p=, 01)$, en sus dimensiones de soledad emocional $(r=-, 22)(p=, 05)$, y social $(r=-, 38)(p=, 01)$ así como con el descontrol emocional $(r=-, 26)(p=, 01)$. Para este grupo de mujeres tener empleo se asocia a una predicción afectiva elevada en un $52.1 \%$ de modo significativo $\left(\chi^{2}=.024\right)$.

Para el grupo de madres después de reagrupar a sus hijos, los análisis indican que existen relaciones significativas entre la predicción afectiva y la satisfacción con la vida $(r=, 54)$ $(p=0,01)$, la resiliencia $(r=, 23)(p=, 05)$ la autoeficacia $(r$ $=, 24)(p=, 05)$ y la percepción que tienen las madres de la satisfacción de sus hijos $(r=, 35),(p=, 01)$ y negativamente con la soledad $(r=-, 31)(p=, 01)$, tanto emocional $(r=$ $-, 25)(p=, 05)$, como social $(r=-, 31)(p=, 01)$, el estrés $(r$ $=-31)(p=, 01)$, el descontrol $(r=,-27)(p=05)$ y el afecto negativo $(r=-, 33)(p=, 01)$.

$\mathrm{El}$ análisis de regresión lineal paso a paso, explica en el último modelo de regresión formado por 3 pasos $(F(3,88)=$ $9,85 ; \mathrm{p}=, 000)$, un $23.2 \%$ de la variabilidad de la predicción afectiva (Tabla 4). Como se aprecia en los coeficientes de regresión la variable que entró en primer lugar en la ecuación y la más relevante para la explicación de la predicción afectiva antes del reencuentro, fue la soledad social percibida por la madre, que resulta un predictor interesante ya que explica el $15 \%$ de la varianza por sí sola. En el segundo paso, ingresa la variable desempleo (en sentido negativo) aportando un 4 $\%$ de explicación. En tercer lugar, la variable satisfacción con la vida resulta ser un predictor válido, contribuyendo con un $4 \%$ más a la explicación de la variabilidad de la predicción afectiva. De este modo, se comprueba que una menor percepción de soledad social, poseer empleo y mayor 
satisfacción con la vida, explican una mayor predicción afectiva positiva antes del encuentro.

Se realiza un análisis de regresión lineal paso a paso de la predicción afectiva para el grupo de madres después de la reagrupación con los hijos (Tabla 5). De este modo, se comprueba que el último modelo se forma con 3 pasos $(\mathrm{F}(3.27)=9.92 ; p=, 000)$, explicando un $47 \%\left(\mathrm{R}^{2}=, 52\right.$; $\left.\mathrm{R}^{\text {2corregida }}=, 47\right)$ de la variabilidad de la predicción afectiva. La variable que entró en primer lugar en la ecuación fue la satisfacción con la vida de la madre $(\beta=, 291 ; t=2,40 ; p$ $=, 023)$, que explica por sí sola un $33 \%$. La segunda variable en entrar en la ecuación fue la percepción de satisfacción del hijo en País Vasco $(\beta=, 334 ; t=2,57 ; p=, 016)$, que explica significativamente un $7 \%$ más y por último, entró la percepción de descontrol emocional que explica un $7 \%$ más $(\beta=-, 487 ; \mathrm{t}=-2,22 ; \mathrm{p}=, 035)$. De este modo, se comprueba que la mayor satisfacción vital de la madre, la satisfacción percibida de los hijos, y la menor percepción de descontrol emocional, son las variables que mejor explican la predicción afectiva positiva en el grupo de después del evento del reencuentro.

Los resultados muestran que la predicción afectiva antes y después de la reagrupación familiar mantiene valores elevados, siendo una variable que favorece positivamente el proceso. Con el fin de conocer los posibles efectos amortiguadores de la predicción en las variables que la explican, presentamos el análisis de mediación basado en el sistema macro de SPSS (Preacher y Hayes, 2004), que a partir del test de Sobel determina si el efecto indirecto de los mediadores es significativamente distinto de cero. Si el intervalo de confianza no incluyera el 0 , entonces el efecto es significativo y la mediación existe (Preacher y Hayes, 2004).

El primer modelo hipotético de mediación, utiliza las variables satisfacción con la vida y soledad social, explicativas de la predicción afectiva para el grupo de mujeres antes del reencuentro. De este modo se prueba en primer lugar un modelo donde $\mathrm{X}$ (variable causa) es la satisfacción con la vida, M (mediador) es la predicción afectiva antes de la

Tabla 4. Resumen del modelo: predicción afectiva de la reagrupación familiar antes del reencuentro

\begin{tabular}{lcccccccccc}
\hline & & & & & & & & \multicolumn{3}{c}{ Estadísticos de cambio } \\
\cline { 7 - 11 } Modelo & $\mathrm{R}$ & $\mathrm{R}^{2}$ & $\begin{array}{c}\mathrm{R}^{2} \\
\text { corregida }\end{array}$ & $\mathrm{F}(\mathrm{gl})$ & $\mathrm{P}<$ & Error típ. Estimación & $\begin{array}{c}\text { Cambio } \\
\mathrm{R}^{2}\end{array}$ & $\begin{array}{c}\text { Cambio } \\
\text { en } \mathrm{f}\end{array}$ & $\mathrm{Gl}(1,2)$ & $\mathrm{P}<$ \\
\hline $1 . \mathrm{a}$ & $.402^{\mathrm{a}}$ & .162 & .152 & $16.78(1)$ & .000 & 1.872 & .162 & 16.785 & 1.87 & .000 \\
$2 . \mathrm{b}$ & $.461^{\mathrm{b}}$ & .212 & .194 & $11.59(2)$ & .000 & 1.825 & .051 & 5.527 & 1.86 & .021 \\
$3 . \mathrm{c}$ & $.508^{\mathrm{c}}$ & .258 & .232 & $9.85(3)$ & .000 & 1.782 & .046 & 5.225 & 1.85 & .025 \\
\hline
\end{tabular}

Variable dependiente: predicción afectiva antes de la reagrupación familiar

a. Variables predictoras: soledad social

b. Variables predictoras: soledad social, desempleo

c. Variables predictoras: soledad social, desempleo, satisfacción con la vida

Tabla 5. Resumen del modelo: predicción afectiva después reagrupación familiar

\begin{tabular}{|c|c|c|c|c|c|c|c|c|c|c|}
\hline \multirow[b]{2}{*}{ Modelo } & \multirow[b]{2}{*}{$\mathrm{R}$} & \multirow[b]{2}{*}{$\mathrm{R}^{2}$} & \multirow[b]{2}{*}{$\mathrm{R}^{2}$ corregida } & \multirow[b]{2}{*}{ a F(gl) } & \multirow[b]{2}{*}{$\mathrm{P}<$} & \multirow[b]{2}{*}{$\begin{array}{l}\text { Error típ. } \\
\text { Estimación }\end{array}$} & \multicolumn{4}{|c|}{ Estadísticos de cambio } \\
\hline & & & & & & & $\begin{array}{c}\text { Cambio } \\
\mathrm{r}^{2}\end{array}$ & $\begin{array}{c}\text { Cambio } \\
\mathrm{f}\end{array}$ & $\mathrm{Gl}(1,2)$ & $\mathrm{P}<$ \\
\hline 1.a & $.594^{\mathrm{a}}$ & .353 & .330 & $15.79(1)$ & .000 & .839 & .353 & 15.793 & 1.29 & .000 \\
\hline $2 . b$ & $.661^{b}$ & .437 & .397 & $10.87(2)$ & 000 & .796 & .085 & 4.214 & 1.28 & .050 \\
\hline 3.c & $.724^{\mathrm{c}}$ & .525 & .472 & $9.92(3)$ & .000 & .745 & .087 & 4.953 & 1.27 & .035 \\
\hline
\end{tabular}

Variable dependiente: predicción afectiva después de reagrupados

a. Variables predictoras: satisfacción con la vida madre

b. Variables predictoras: satisfacción con la vida, satisfacción con la vida percibida de su hijo

c. Variables predictoras: satisfacción con la vida, satisfacción con la vida percibida de su hijo, descontrol emocional 
reagrupación familiar, e Y (variable resultado) es la soledad social (Figura 1). Además, indican que el efecto directo de la satisfacción en la soledad social es significativo y negativo $(\beta=-, 289, \mathrm{t}=-5,91, p=, 000)$. El efecto de la satisfacción en la predicción es significativo y positivo $(\beta=, 486, \mathrm{t}=3,84$; $p=, 000)$. El efecto de predicción en la soledad social es negativo y significativo $(\beta=-.090, t=-2,37, p=, 019)$. De este modo, el efecto indirecto de la mediación también es significativo explicando un $4,4 \%$ de la varianza $(\beta=-, 044$; IC 95\% [-,0882, -,0003]). Los resultados señalan que la satisfacción mediante la predicción afectiva amortigua la percepción de soledad social $(\beta=-, 245 t=-4,78 ; p=, 000)$. Sin embargo, se trata de una mediación parcial (Hayes, 2013), ya que sigue siendo significativa la relación negativa entre satisfacción con la vida y soledad social.

En el caso del grupo madres después de reagrupar, se utiliza igualmente las variables explicativas de la predicción afectiva como son satisfacción con la vida y descontrol emocional. La satisfacción percibida del hijo no correlaciona, ni con la satisfacción con la vida de la madre $(\mathrm{r}=, 30)$ $(p=, 06)$, ni con su percepción de descontrol $(\mathrm{r}=-, 03)(p=$ $, 82)$ lo que indica que no hay relaciones entre ellas. Si se prueba un modelo en donde se toma como variable causa $\mathrm{X}$, el descontrol emocional, como mediadora $\mathrm{M}$, la predicción afectiva después del reencuentro, y como resultado $\mathrm{Y}$, la satisfacción con la vida. El modelo de mediación (Figura 2) arroja como resultado lo siguiente: el efecto directo del descontrol en la satisfacción es negativo y significativo $(\beta$ $=-, 585, t=-2,94 ; p=, 004)$. El efecto del descontrol en la predicción es negativo y significativo $(\beta=-, 418, t=-2,75$, $p=, 007)$. El efecto de la predicción en la satisfacción es positivo y significativo $(\beta=, 623, t=4,62 ; p=, 000)$. Por lo tanto, el efecto indirecto de la mediación (figura 2) es significativo y explica un $26 \%$ de la varianza $(\mathrm{B}=-, 26$; IC $95 \%[-4810,-0415])$. La predicción afectiva ejerce un efecto mediador completo (Hayes, 2013) en la relación entre el

Figura 1. Modelo de Mediación simple de la predicción afectiva antes del reencuentro sobre la relación de la satisfacción con la vida y la soledad social

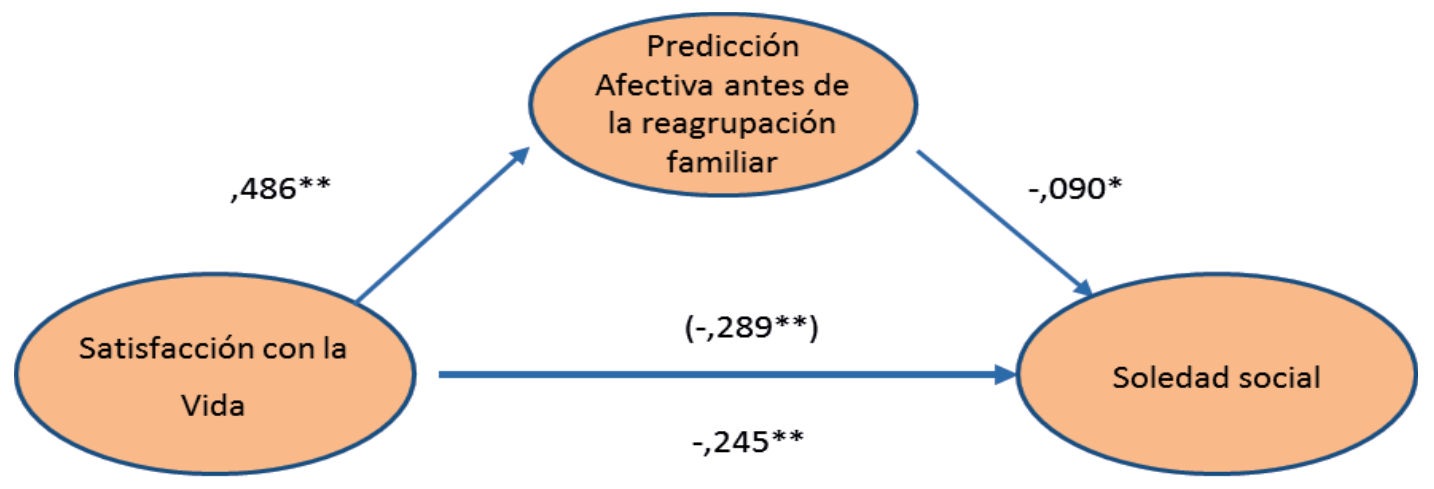

Nota. Se presentan los coeficientes de regresión B no estandarizados.

El número entre paréntesis indica el valor beta antes de incluir las variables mediadoras **p $<, 01 ; * \mathrm{p}<, 05$.

Figura 2. Modelo de Mediación simple de la predicción afectiva después del reencuentro sobre la relación entre el descontrol emocional y la satisfacción con la vida

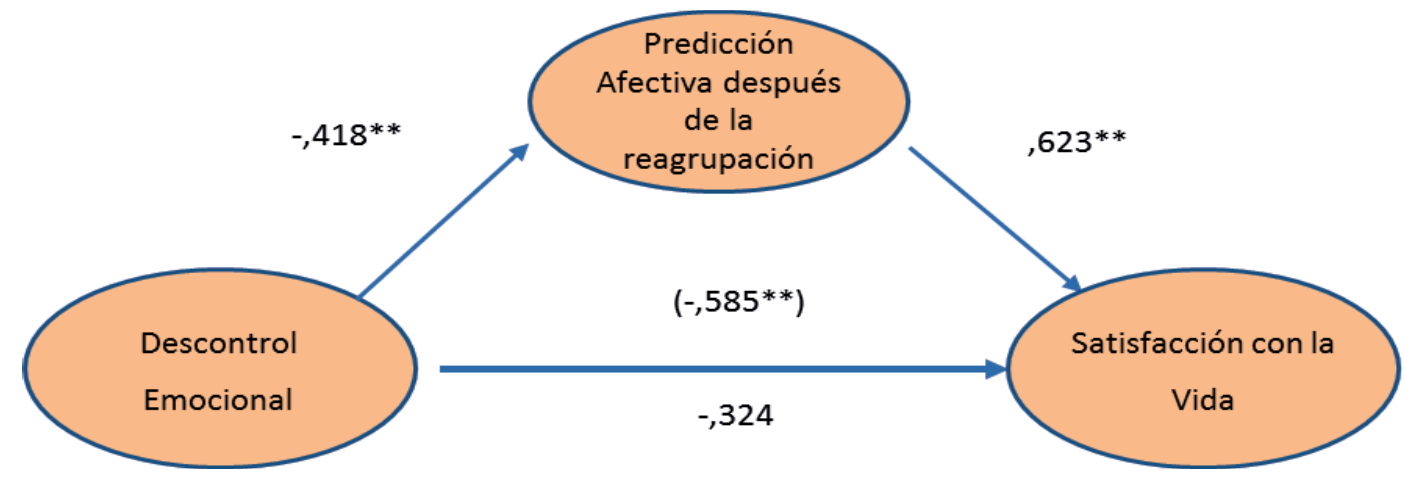

Nota: Se presentan los coeficientes de regresión B no estandarizados.

El número entre paréntesis indica el valor beta antes de incluir las variables mediadoras. ${ }^{* *} p<, 01 ; * p<, 05$ 
descontrol emocional y la satisfacción con la vida porque una vez introducida la variable mediadora la relación deja de ser significativa. Se evidencia un menor descontrol emocional y mayor satisfacción con la vida mediante la predicción afectiva $(\beta=-, 324, t=-1,75, p=, 083)$.

\section{Discusión}

Los resultados confirman la hipótesis de que el grupo de madres transnacionales en situación previa a reagrupar a sus hijos tienden a realizar una predicción afectiva positiva y sobrevalorada, de forma tal que la intensidad y duración de las emociones previstas antes del evento son más elevadas de un modo estadísticamente significativo que en las mujeres después de haber reagrupado. Esto concuerda con los principios del modelo del "affective forecasting" (Wilson y Gilbert, 2003) en el que una vez acontecida la experiencia emocional de facto se reduce la sobrevaloración de la misma. Sin embargo, aunque hay diferencias significativas en la percepción de satisfacción, de los afectos, la resiliencia, la evitación, la soledad y el estrés entre las dos condiciones de reunificación familiar en intensidad y duración, la predicción afectiva, menor en valencia en el grupo después, sigue siendo positiva y observa una influencia específica. Esto implica la percepción de un coste emocional aceptable, aunque a veces perciban descontrol, lo que no impide un augurio ilusionado a futuro aunque sea en menor medida que en la situación previa a la reunión con los hijos. Esto sugiere que la ilusión y las expectativas generadas en el proceso antes de la reagrupación influye en las respuestas emocionales posteriores al evento real manteniéndose de modo positivo. Así, los resultados muestran que las personas que predicen positivamente un suceso, tienden a confirmar sus estimaciones en la misma dirección.

Podemos decir que las personas que predicen positivamente un suceso, tienden a confirmar sus estimaciones en la misma dirección y se reafirma como claro indicador hacia el logro, como facilitador de procesos adaptativos (Taylor, 1991) y de emociones positivas en tanto disminuyen la posibilidad de elementos negativos o depresivos, mejorando la salud (Taylor y Brown, 1988) y el bienestar del proceso de reunificación familiar con los hijos. De este modo podemos observar una especie de profecía que se auto cumple (Wilson y Gilbert, 2003). La asimilación entre lo esperado y lo real facilita la cercanía entre las expectativas y la realidad, o entre el antes y el después del evento.

Los resultados corroboran que la predicción afectiva del reencuentro (Gilbert, 2006), junto con la satisfacción vital y la balanza de afectos positivos asociado a la vida en cercanía con los hijos son coherentes con el bienestar subjetivo, que se mantiene más o menos estable a lo largo de la vida (Javaloy, 2007). Las mujeres latinoamericanas determinadas por una cultura proclive a la familia (Ayón et al., 2010), donde los hijos se asocian a felicidad (Pedone, 2003) sostienen niveles elevados y constantes de bienestar, al igual que se comprueba en otros estudios (Peña-Pinzón, Gómez-Berrocal y Fernández-Parra, 2014). La llegada de los hijos moviliza estructuras, confiere una sensación positiva de realización y apoyo, que mantiene estable la satisfacción con la vida (Hernández, Pozo, Alonso y Martos, 2005).

No obstante, el reencuentro familiar transnacional después de tiempo separados genera tensión en las dinámicas emocionales, familiares, y sociales (Offenhenden, 2013) que explica la merma de apreciación de resiliencia, pues disminuye la percepción de autoeficacia, de sentido y significado de la reagrupación como variable operativa según predecía el modelo de Wilson y Gilbert (2003). Así, las mujeres después de reagrupar hijos, presentan mayores niveles de estrés y más descontrol emocional (Wilson y Gilbert, 2003) indicando que en el proceso de reencuentro familiar se deben renegociar espacios y vínculos con los hijos a fin de fortalecer la cohesión familiar (Gil y Vega, 1996), lo que se asocia al aumento del grado de estrés en el grupo de mujeres ya en convivencia con su prole (Zarza González y Sobrino Prados, 2007). Esta emergencia de estrés se vincula a peor salud psicológica, mental y social (Wlodarczyk, Basabe,, Páez, Amutio, A, García, Reyes”y Villagrán, 2016), lo que muestra las dificultades emocionales que tienen las mujeres en el proceso de reagrupación (Weiss, 1972). Por eso consideramos una aportación de este estudio señalar que el coste emocional no es tan elevado como se esperaba y muestra a mujeres con capacidades y competencias personales, sociales, y transculturales desarrolladas en el contexto migratorio que mantienen la proyección de futuro positiva. Así mismo, mantienen respuestas de autoeficacia y sentido ante las adversidades de la realidad de un proyecto que favorece el ajuste social, psicológico y vital (Sobral, Gómez-Fraguela, Romero, Luengo y Villar, 2012) como es la reagrupación familiar transnacional.

La presencia de soledad, producto de la migración, de una menor red social y emocional se manifiesta con mayor facilidad en el género femenino (Golden et al., 2009). Esta realidad se compensa con valores culturales de estrecha vinculación familiar (Ayón et al., 2010) que se mantienen en la distancia, Es por eso que el reencuentro aporta la restitución de los vínculos de apego y el apoyo social (Rivera, 2007), reduciendo el malestar que produce la separación Así se 
explica que las mujeres reagrupadas con sus hijos muestren de un modo estadísticamente significativo, menos percepción de soledad, emocional y social, y que el reencuentro familiar aporte elementos positivos afines a los imaginados. Se esperaba que los afectos negativos fuesen mayores después de reunidos debido a la inestabilidad, la presión social y cultural (Elgorriaga, Arnoso, Martínez-Taboada, y Otero, 2012), sin embargo, las emociones negativas se neutralizan en la balanza de afectos asociadas a indicadores de bienestar (Diener y Lucas, 1999).

El hecho de que la predicción afectiva correlacione en positivo con variables de ajuste psicosocial antes y después de reagrupar, señala su potencial favorable. El análisis de regresión indica que cuanto menor sea la percepción de soledad social de una madre separada de sus hijos, mayor valoración positiva hará de su vida (Diener et al., 1985). Estos resultados son coherentes en tanto las madres latinoamericanas se mueven en redes de apoyo social (Pedone, 2003) que facilitan su proceso de reencuentro familiar. Contar con estos apoyos disminuye sus vivencias de soledad (Golden et al., 2009) y garantiza una red de contención para sus hijos. A esto se suma que la mujer se reafirma en sus decisiones de reencuentro, en tanto el tener un trabajo le permite gestionar ahorros y condiciones para recibir a sus hijos (Castaneda., Felt., Martinez-Taboada, y Castañeda, 2013).

Comprobamos que la predicción afectiva antes del reencuentro, media entre la satisfacción vital y la soledad social. Estos resultados tienen sentido ya que la proyección e ilusión del reencuentro aporta satisfacción a las madres que se encuentran alejadas de sus hijos, producen bienestar aún antes de experimentarlo directamente con una "anticipación de la satisfacción" (Gilbert, 2006), y contraria a la percepción de soledad. Por su parte, para el grupo de madres que ya han reagrupado a sus hijos, la satisfacción con la vida de la madre y del hijo, junto con el descontrol percibido de la madre en negativo, explican la predicción afectiva positiva después del reencuentro.

Para las mujeres latinoamericanas, con valores impregnados por una cultura colectivista (Ayón et al., 2010;) donde la madre cumple un rol fundamental (Gissi, Zubieta y Páez, 2002), los hijos se asocian a principios interiorizados que refuerzan la percepción de satisfacción con la vida en el ámbito familiar (Diener, 1984), e influye en el aumento de la vivencia emocional congruente y positiva después de reagrupados. Los resultados indican que en tanto se percibe menor descontrol emocional ante la presencia de los hijos, mejor es la regulación emocional (Hervás y Jodar, 2008), lo que favorece positivamente el reencuentro.
Estudios previos indican que la predicción afectiva debe ajustarse a la realidad para garantizar la capacidad de adaptación a las situaciones, favorecer el control psicosocial (Morling y Evered, 2006), el bienestar y la salud (Garrido, Guzmán; Santelices, Vitriol, y Baeza 2015). El análisis de mediación evidencia que la predicción afectiva con valencia e intensidad positiva se relaciona negativamente con el descontrol emocional y positivamente con una mayor satisfacción con la vida. Es por ello que consideramos que las predicciones afectivas positivas son un factor de protección en el control de emociones negativas (Buehler, McFarland, Spyropoulos y Lam, 2007), lo que se vincula a una mejor salud general y bienestar. (Taylor y Brown, 1988) en la situación posterior al evento de la reagrupación familiar transnacional.

En resumen, la predicción afectiva positiva favorece la regulación y el control de los afectos en los dos grupos de madres antes y después de la reagrupación con los hijos y se vincula a bienestar (Gilbert, 2006), resiliencia y diversos grados de estrés, con diferencias significativas entre ellos pero en la misma dirección positiva. Además, la predicción afectiva amortigua la relación entre la soledad social y la satisfacción con la vida antes del evento, y el descontrol emocional y la satisfacción vital después de la reagrupación (Martínez-Taboada y Otero, 2015).

Señalar que profundizar en el modo de combatir los desajustes con la realidad (Kermer, Driver-Linn, Wilson y Gilbert, 2006) contribuiría a fortalecer la predicción afectiva positiva que se puede incrementar con métodos como el mindfulness para mejorar el pronóstico afectivo de estas mujeres (Emanuel, Updegraff, Kalmbach, y Ciesla, 2010), convirtiéndose en un mecanismo socio-cognitivo protector (Willner-Reida, Smith, Borden, y MacLeoda, 2012) muy diferente de la idealización definida como mecanismo de defensa de corte subjetivo psicodinámico e individual.

Por último, en línea con el modelo del "affective forecasting" de Wilson y Gilbert $(2003,2006)$ podemos decir que la predicción afectiva de valencia positiva, de intensidad y duración variable entre el antes y después de la reagrupación familiar transnacional fortalece y protege el proceso socio-cognitivo de la reunificación el cual se vería favorecido si se asocia a políticas (Checa y Montserrat, 2014; Urzúa, Vega, Jara, Trujillo, Muñoz, y Caqueo-Urizar, 2015) que mejoren la integración y calidad de vida de las familias trasnacionales. 


\section{Referencias}

Achotegui, J. (2004). Emigrar en situación extrema: el Síndrome del inmigrante con estrés crónico y múltiple (Síndrome de Ulises). Revista Norte de Salud Mental, 21, 39-52.

Ayón, C., Marsiglia, F. F., y Bermúdez-Parsai, M. (2010). Latino family mental health: Exploring the role of discrimination and familismo. Journal of Community Psychology, 38, 742-756.

Blanco, A., y Díaz, D. (2005). El bienestar social, su concepto y medición. Universidad autónoma de Madrid. Psicothema, 17 (4), 582-589.

Bonanno, G. (2004). Loss, trauma and human resilience. Have we underestimated the human capacity to thriver after adversity events? American Psychologist, 59 (1), 20-28.

Borges, A., Prieto,P., Ricchetti,G., Hernández-Jorge,C., y RodríguezNaveiras, E. (2008). Validación cruzada de la factorización del Test UCLA de Soledad. Psicothema, 20, 924-927.

Buehler, R., McFarland, C., Spyropoulos, V., y Lam, C. K. (2007). Motivated Prediction of Future Feelings: Effects of Negative Mood and Mood Orientation on Affective Forecasts. Personality and Social Psychology Bulletin, 33, 1265-1278.

Cabañero, M., Martínez, M., Cabrero, J., Orts, M., Reig, A, Reig, R., y Tosal, B. (2004). Fiabilidad y validez de la Escala de Satisfacción con la Vida de Diener en una muestra de mujeres embarazadas y puérperas. Psicothema, 16, 448-45.

Campbell, D., y Stanley, J. (2011). Diseños experimentales y cuasiexperimentales en la investigacion social. Amorrortu Editores. Buenos Aires. Argentina.

Campos, M., Iraurgui, J., Páez , D., Velasco, C. (2004). Afrontamiento y regulación emocional de hechos estresantes. Un metanálisis de 13 estudios. Boletín de Psicología, 82, 25-44.

Castaneda, X., Martinez-Taboada, C., Felt. E., Castaneda, N. (2013): Socioeconomic Challenges and Cultural Psychology: Processes of Acculturation. En L. Sher (Ed.), Immigration and Mental Health Psychiatric disorders and suicidal behaviour among immigrants and refugees, (pp.67-84). New York: Nova.

Carlsmith, K. M., Wilson, T. D.,y Gilbert, D. T. (2008). The paradoxical consequences of revenge. Journal of Personality and Social Psychology, 95, 1316-1324.

Checa, J. C., y Monserrat, M. (2015). La integración social de los hijos de inmigrantes africanos, Europeos del Este y latinoamericanos. Un estudio de caso en España. Universitas Psychologica, 14, 475-486.

Cohen, S., Kamarack, T., y Mermelstein, R. (1983). A global measure of perceived stress. Journal of Health and Social Behavior, 24, 385-396.

Cyrulnik, B. (2002). Los patitos feos. La resiliencia: una infancia infeliz no determina la vida. Madrid: Gedisa.

Diener, E. (1984). Subjective well-being. Psychological Bulletin, 95, 542-575.

Diener, E., Emmons, R. A., Larsen, R. J., y Griffin, S. (1985). The Satisfaction With Life Scale. Journal of Personality Assessment, 49, 71-75.

Diener, E., y Lucas, R. E. (1999). Personality and sujective well-being. En D. Kahneman, E. Diener y N. Schwarz (Eds), Well-being: The foundations of hedonic psychology (pp. 213-227). New York: Russell Sage Fundation.

Elgorriaga, E., Martínez-Taboada, C., y Arnoso, A. (2014): La importancia del género y el origen cultural en los procesos migratorios y en la satisfacción con la vida de la población inmigrante. ISSN: 0212-8179. Boletín de Psicología, 112, 7-34.

Elgorriaga, E., Arnoso, A., Martínez-Taboada, C., y Otero, N. (2012): Proceso migratorio y ajuste psicológico de las mujeres latino americanas y magrebíes: un análisis desde la perspectiva de género. Migraciones.

Emanuel, A. S., Updegraff, J. A., Kalmbach, D. A., y Ciesla, J. A. (2010). The role of mindfulness facets in affective forecasting. Personality and Individual Differences, 49, 815-818

Fernandes de Araújo, L., y Bermúdez, M.P. (2015) Resiliencia en adultos: una revisión teórica. Terapia Psicológica, 33, 257-276.

Fontes, S., García, C. Garriga, A.J., Pérez-Llantada, M.C., y Sarri, E. (2010). Diseños de investigación en Psicología. UNED: Madrid.
Garrido, L., Guzmán, M., Santelices, M. P. Vitriol, V., y Baeza, E. (2015). Estudio comparativo de los estilos de apego adulto en un grupo de mujeres con y sin diagnóstico de depresión. Terapia Psicológica, 33, 285-295

Gil, A. G., y Vega, W. A. (1996). Two different worlds: acculturation stress and adaptation among Cuban and Nicaraguan families. Journal of Social and Personal Relationships, 13, 435-456.

Gilbert, D. (2006). Tropezar con la felicidad. Barcelona: Destino.

Gilbert, D. T., y Wilson, T. D. (2009). Why the brain talks to itself: Sources of error in emotional prediction. Philosophical Transactions of the Royal Society, 364, 1335-1341.

Gissi, J., Zubieta, E., y Páez, D. (2002). Cultura y Valores: la identidad social y cultural de América Latina. En J. F. Morales, D. Páez, A. L Kornblit, y D. Asún (Coords). Psicología Social (pp. 57-94). Buenos Aires: Prentics Hall.

Golden, J., Conroy, R.M, Bruce, I., Denihan, A., Greene, E., Kirby, M., y Lawlor, B. A. (2009). Loneliness, social support networks, mood and wellbeing in community-dwelling elderly. International Journal of Geriatric Psychiatry, 24, 694-700.

Graham, S. V. (2010). Transnational motherhood: The impact of immigration related mother-child separation on Latina mothers. (Tesis doctoral) Columbia University. Recuperado desde http://search.proquest.com/ docview/858611485

Gratz, K. L., y Roemer, L. (2004). Multidimensional assessment of emotion regulation and dysregulation: Development, factor structure, and initial validation of the difficulties in emotion regulation scale. Journal of Psychopathology and Behavioral Assessment, 26, 41-54.

Hayes, A. F. (2013). Introduction to mediation, moderation, and conditional process analysis. A regression-based approach. London: The Guildford Press.

Hernández, S., Pozo, C., Alonso, E. y Martos, M.J. (2005). Estructura y funciones del apoyo social en un colectivo de inmigrantes marroquíes. Anales de Psicología, 21, 304-315.

Hervás G., y Jodar, R. (2008). Adaptación al castellano de la Escala de Dificultades en la Regulación Emocional. Clínica y Salud, 19, 139-156.

Javaloy, F. (2007). Bienestar y felicidad de la juventud española. INJUVE Madrid: Ministerio de trabajo y asuntos sociales.

Kermer, D. A., Driver-Linn, E., Wilson, T. D., y Gilbert, D. T. (2006). Loss aversion is an affective forecasting error. Psychological Science, $17,649-653$.

Lemos, M., y Londoño, N. H. (2006). Construcción y validación del cuestionario de dependencia emocional en población colombiana. Acta Colombiana de Psicología, 9, 127-140.

Martinez-Taboada, C., y Otero, N. (2015). Communication and Interaction in a Group of Sub-Saharan Africa Immigrant women. En S.S. Fehr (Ed.), 101 Interventions in Groups II (pp.76-82). Abingdon UK: Routdlege Press.

Martínez-Taboada, C., Arnoso, A., y Elgorriaga, E. (2006). Estudio del Choque Psicosocial de la población inmigrada a Donostia. San Sebastián: Ayuntamiento de San Sebastián.

Medina Villegas, M. C. (2011). Los ausentes están siempre presentes: una aproximación interpretativa de la experiencia maternofilial transnacional entre España y Colombia. (Tesis doctoral) Universidad Complutense de Madrid. Recuperado desde http://eprints.ucm.es/12191/1/T32120.pdf

Molina, T., Gutiérrez, A. G., Hernández, L. y Contreras, C. (2008). Estrés psicosocial: algunos aspectos clínicos y experimentales. Anales de Psicología, 24, 353-360.

Montecino, S. (1999). Identidades de género en América Latina. El lenguaje de la diversidad. En M. A. Garretón (Ed.), América Latina: un espacio cultural en el mundo globalizado. Debates y perspectivas (pp. 249-293). Bogotá: Convenio Andrés Bello.

Moreno-Jiménez, M. P., y Hidalgo, M. C. (2011). Medición y predicción de la satisfacción con la vida en inmigrantes empleados en España. Diferencias según su situación administrativa. Anales de Psicología, 27 (1), 179-185

Morling, B., y Evered, S. (2006). Secondary control reviewed and defined. Psychological Bulletin, 132, 269.

Nielsen, L., Knutson, B., y Carstensen, L. L. (2008). Affect Dynamics, Affective Forecasting, and Aging. Emotion, 8, 318-330. 
Offenhenden, M. (2013). Cuerpos para el trabajo. Una mirada sobre la gestión de los trastornos de la salud de las trabajadoras domésticas migrantes. Arxiu d'Etnografia de Catalunya, 13, 137-159.

Pedone, C. (2003). Tú siempre jalas a los tuyos. Cadenas y redes migratorias de las familias ecuatorianas hacia España. (Tesis doctoral), Universidad autónoma de Barcelona. Recuperado desde http://ddd.uab. cat/pub/tesis/2004/tdx-1027104-170605/cp1de5.pdf

Peña-Pinzón, J. A., Gómez-Berrocal, C., y Fernández-Parra, A. (2014). El papel de la categorización social y las diferencias sociodemográficas en la autoestima, el ajuste y el bienestar psicológicos de una muestra de inmigrantes. Anales de Psicología, 30, 560-572.

Pérez Grande, M. D. (2008). Mujeres inmigrantes, realidades, estereotipos y perspectivas educativas. Revista Española de Educación Comparada 14, 137-175.

Preacher, K. J., y Hayes, A. F. (2004). SPSS and SAS procedures for estimating indirect effects in simple mediation models. Behavior Research Methods, Instruments, \& Computers, 36(4), 717-731. doi: 10.3758/BF03206553

Remor, E., y Carrobles, J.A. (2001). Versión Española de la escala de estrés percibido (PSS-14): Estudio psicométrico en una muestra VIH+. Ansiedad y Estrés, 7, 195-201.

Rivera, F. I. (2007). Contextualizing the experience of young latino adults: acculturation, social support and depression. Journal of Immigrant and Minority Health, 9, 237-244.

Rodríguez, M., Pereyra, M. G., Gil, E., Jofré, M., De Bortoli, M., y Labiano, L. M. (2009) Propiedades psicométricas de la escala de resiliencia versión argentina. Evaluar, 9, $72-82$.

Russell, D., Peplau, L. A., y Cutrona, C.E. (1980). The revised UCLA Loneliness Scale: Concurrent and discriminant validity evidence. Journal of Personality and Social Psychology, 39, 472-480.

Sandín, B., Chorot Raso, P., Lostao, L., Joiner, T., Santed Germán, M., y Valiente, R. (1999). Escala PANAS de afecto positivo y negativo: validación factorial y convergencia transcultural. Psicothema, 11, 37-51.

Sandín, B., y Chorot, P. (2003). Cuestionario de afrontamiento del estrés (CAE): desarrollo y validación preliminar. Revista de Psicopatología y Psicología Clínica, 8, 39-54

Sobral, J., Gómez-Fraguela, J.A., Romero, E., Luengo, E., y Villar, P. (2012). Riesgo y protección de desviación social en adolescentes inmigrantes: Personalidad, familia y aculturación. Anales de Psicología, 2, 664-674.

Taylor, S. (1991). Seamos optimistas. Barcelona: Martinez Roca.
Taylor, S. E., y Brown, J. D. (1988). Illusion and well-being: A social psychological perspective on mental health. Psychological Bulletin, 103, 193-210.

Urzúa, A., Vega, M., Jara, A., Trujillo, S., Muñoz, R., y Caqueo-Urízar, A. (2015). Calidad de vida percibida en inmigrantes sudamericanos en el norte de Chile. Terapia Psicológica, 33(2), 139-156.

Vitar, B. (2012). Mujeres Latinoamericanas en Madrid. Expectativas y negociaciones en sus trayectorias migrantes Naveg@mérica Revista electrónica de la Asociación Española de Americanistas, 8. Recuperado desde http://www.plataformademocratica.org/Publicacoes/20662.pdf

Wagnild, G., y H. Young. (1993): Development and psychometric: evaluation of the resilience scale. Journal of Nursing Measurement, l(2), 165-178.

Watson, D., Clark, L. A., y Tellegen, A. (1988). Development and validation of brief measures of positive and negative affect: the PANAS scales. Journal of Personality and Social Psychology, 54, 1063.

Weiss, J. M. (1972). Psychological factors in stress and disease. Scientific American, 226, 104-113.

Willner-Reida, J, Smith, N., Bowden, H., y MacLeoda, A. (2012). Affective forecasting in problem gamblers. International Gambling Studies, 12, 295-307.

Wilson, T., y Gilbert, G. (2003). Affective Forecasting. En L. Berkowitz y M. P. Zanna (Eds). Advances in Experimental Social Psychology (pp. 345-412). Chicago: Academic Press.

Wilson, T. D., \& Gilbert, D. T. (2005). Affective forecasting knowing what to want. Current Directions in Psychological Science, 14(3), 131-134.

Wlodarczyk, A., Basabe, N, Páez, D., Amutio, A., García, F., Reyes" C. y Villagrán, L. (2016). Positive Effects of Communal Coping in the Aftermath of a Collective Trauma: The Case of 2010 Chilean Earthquake. European Journal of Education and Psychology, 9, 9-19. doi: 10.1016/j. ejeps.2015.08.001

Zarza González, M. J., y Sobrino Prados, M. I. (2007). Estrés de adaptación sociocultural en inmigrantes latinoamericanos residentes en Estados Unidos vs. España: Una revisión bibliográfica. Anales de Psicología, 23, 72-84. 
